\title{
Outcome-specific conditioned inhibition in Pavlovian backward conditioning
}

\author{
ANDREW R. DELAMATER \\ Brooklyn College and the Graduate School of the City University of New York, Brooklyn, New York \\ VINCENT M. LOLORDO \\ Dalhousie University, Halifax, Nova Scotia, Canada \\ and \\ WENDY SOSA \\ Brooklyn College and the Graduate School of the City University of New York, Brooklyn, New York
}

\begin{abstract}
In the present experiments, the outcome specificity of learning was explored in an appetitive Pavlovian backward conditioning procedure with rats. The rats initially were administered Pavlovian backward training with two qualitatively different unconditioned stimulus conditioned-stimulus (US-CS) pairs of stimuli (e.g., pellet $\rightarrow$ noise or sucrose $\rightarrow$ light), and then the effects of this training were assessed in Pavlovian-to-instrumental transfer (Experiment 1) and retardation-of-learning (Experiment 2) tests. In the transfer test, it was shown that during the last 10-sec interval, the CSs selectively reduced the rate of the instrumental responses with which they shared a US, relative to the instrumental responses with which they did not share a US. The opposite result was obtained when the USs (in the absence of the CSs) were presented noncontingently. In the retardation test, conditioned magazine approach, responding to the CSs was acquired more slowly when the stimulus-outcome combinations in the backward and the forward conditioning phases were the same, as compared with when they were reversed. These results are collectively in accord with the view that Pavlovian backward conditioning can result in the formation of outcome-specific inhibitory associations. Alternative views of backward conditioning are also examined.
\end{abstract}

One of the fundamental goals of learning theory has been to characterize the contents of learning. Studies directed toward an analysis of the contents of Pavlovian learning point to the conclusion that a conditioned stimulus (CS) forms an association with stimulus-specific aspects of the unconditioned stimulus (US) or outcome. Evidence favoring this view has come from a growing list of experiments designed to look for outcome-selective effects of US devaluation (e.g., Colwill \& Motzkin, 1994; Delamater \& LoLordo, 1991), intertrial reinforcement (e.g., Delamater, 1995), blocking (e.g., Betts, Brandon, \& Wagner, 1996; Rescorla, 1999), reinstatement (Delamater, 1997), spontaneous recovery (Rescorla, 1997), stimulus compound summation (Watt \& Honey, 1997), and Pavlovian-toinstrumental transfer of control (e.g., Colwill \& Rescorla, 1988; Delamater, 1996; Kruse, Overmier, Konz, \& Rokke, 1983). In the transfer-of-control test, for example, a CS

This research was supported through PSC-CUNY Grant 62690-00-31, awarded to A.R.D. by the Research Foundation of CUNY. It also served as partial fulfillment of the requirements for the degree of M.A. for W.S. at Brooklyn College. Correspondence concerning this article should be addressed to A. R. Delamater, Brooklyn College-CUNY, Psychology Department, 2900Bedford Ave., Brooklyn, NY 11210 (e-mail: andrewd@ brooklyn.cuny.edu). has been shown to exert greater control over an (independently trained) instrumental response when the CS and the instrumental response were associated previously with the same, as opposed to different, outcomes. This selective control appears to demand that stimulus-specific aspects of the US are associated both with the CS and with the instrumental response.

Most of the studies in which outcome-specific effects in Pavlovian conditioning have been examined have explored the contents of learning that result from forward conditioning procedures, where the CS precedes the US on a given conditioning trial. However, recently, a considerable amount of interest has been directed to the study of backward Pavlovian conditioning, where the US precedes the CS (e.g., Cole \& Miller, 1999; McNish, Betts, Brandon, \& Wagner, 1997; Romaniuk \& Williams, 2000; Silva \& Timberlake, 2000; Silva, Timberlake, \& Cevik, 1998; Williams \& Hurlburt, 2000). Learning, in this situation, has been described from very different theoretical orientations as being excitatory, inhibitory, or both excitatory and inhibitory. However, the issue of the contents of such learning has not been assessed thoroughly. Some theoretical approaches to conditioning are explicit in asserting that backward conditioning can be sensory specific in its content. 
The AESOP model of Wagner and Brandon (1989), for example, gives rise to the expectation that under the right conditions, sensory-specific excitatory or inhibitory associations can be formed. This follows from the model's event-processing and learning rule assumptions. In particular, the model asserts that US presentation causes sensory and motivational representations of the US to be processed initially in their own primary activation states (A1), before these representations decay into their respective secondary activation states (A2) and, ultimately, to their inactive states. Excitatory associations are acquired in this model whenever the CS and the US are concurrently processed in their A1 states. Inhibitory associations are assumed to develop whenever there is concurrent processing of the CS in its A1 state and the US in its A2 state. Thus, if the sensory representation of the US is processed predominantly in its A1 state at the time the CS is presented (e.g., in a backward procedure), sensory-specific excitatory associations should develop. On the other hand, if processing of the sensory representation of the US occurs predominantly in the A2 state when the CS is presented, sensory-specific inhibitory associations should develop.

Prior research on backward conditioning has not focused on the possibility of sensory-specific learning. Instead, such research has been concerned with a different aspect of the model-namely, its prediction that with some US-CS intervals, the CS may simultaneously develop excitatory and inhibitory associations with the US. Although it seems reasonably clear from prior research that a backward CS can simultaneously acquire both excitatory and inhibitory properties (e.g., Cole \& Miller, 1999; McNish et al., 1997; Tait \& Saladin, 1986), the sensory specificity of such learning is less clear.

The point of the present research was to explore learning in the backward conditioning procedure in greater detail, using commonly accepted measures of sensory-specific learning. In Experiment 1, the Pavlovian-to-instrumental transfer-of-control test was used to assess the sensoryspecific control by CSs given backward conditioning. Because the results of the transfer test suggested that the stimulus acquires sensory-specific inhibitory properties, in Experiment 2 a sensory-specific retardation-of-acquisition test was used to assess the sensory specificity of learning in the backward procedure.

\section{EXPERIMENT 1}

In the present experiment, we examined whether backward US-CS training in an appetitive-conditioning paradigm with rats enables the CS to exert outcome-selective instrumental control in a transfer test. This was accomplished by first training rats to associate two different CSs with different USs during a backward conditioning phase (i.e., US1 $\rightarrow$ CS1 and US2 $\rightarrow$ CS2). Then the effects of this training were assessed in a transfer test in which CS1 and CS2 were presented on alternate trials while subjects chose between two instrumental actions, one of which had been reinforced earlier with US1 and the other with US2. If outcome-specific excitatory associations have formed between the CSs and their respective USs, the CSs should influence instrumental choice by selectively increasing the rates of the instrumental responses with which they share an outcome. However, if outcome-specific inhibitory associations between CSs and USs have formed, the CSs should influence instrumental choice by selectively decreasing the rates of the instrumental responses with which they share an outcome.

Method
Subjects
Two squads of 16 experimentally naive, male Sprague-Dawley
rats (supplied by Charles River Breeders) were run: one in each of
two replications. The average weights of the rats in the two replica-
tions were 376 and 488 g, respectively, at the beginning of the exper-
iment. The rats were housed individually in a colony room that was on
a 16:8-h light:dark cycle, and they were maintained at $85 \%$ of their
ad-lib body weights by daily supplemental feedings (given after the
experimental session). The rats in the second replication were run be-
ginning approximately 3 weeks after the first replication had been
completed. The subjects were run in the experiment during the light
phase of their light:dark cycle.

\section{Apparatus}

The apparatus consisted of eight identical standard conditioning chambers, each of which was housed in a sound- and light-resistant shell. The conditioning chambers measured $30.5 \times 24.0 \times 25.0 \mathrm{~cm}$. Two end walls were constructed of aluminum, and the side walls, as well as the ceiling, were made from clear Plexiglas. The floor consisted of $0.60-\mathrm{cm}$-diameter stainless steel rods spaced $2.0 \mathrm{~cm}$ apart. In the center of one end wall $1.2 \mathrm{~cm}$ above the grid floor was a recessed food magazine measuring $3.0 \times 3.6 \times 2.0 \mathrm{~cm}$ (length $\times$ width $\times$ depth). A single 45 -mg pellet (P. J. Noyes Co., Formula A) was dropped onto the magazine floor when this reinforcer was scheduled. A $0.2-\mathrm{ml}$ droplet of a $24 \%$ sucrose solution (w/v) was delivered through a gravity-feed valve (ASCO Red-Hat valve) directly into a well located on the floor of the food magazine when this reinforcer was scheduled. On the inner walls of the recessed magazine were an infrared detector and an emitter enabling the automatic recording of head movements inside the magazine. These were located $0.9 \mathrm{~cm}$ above the floor and recessed $0.8 \mathrm{~cm}$ from the front wall. Located $3.0 \mathrm{~cm}$ to the right of the magazine and $8.0 \mathrm{~cm}$ above the floor was a lever ( $4 \mathrm{~cm}$ in width). This lever protruded into the chamber at all times, but access to the lever was prevented during magazinetraining and Pavlovian phases by a sheet metal covering. Located approximately $3 \mathrm{~cm}$ to the left of the magazine and about $3 \mathrm{~cm}$ away from the front wall was a chain suspended through the ceiling from a microswitch mounted on the outer part of the ceiling. This chain was withdrawn from the chamber during magazine-training and Pavlovian phases. A 6-W light bulb was mounted on the bottom of the side wall of the outer chamber, below and behind the rear wall of the conditioning chamber. When activated, this light bulb remained continuously illuminated. A speaker was mounted approximately $22 \mathrm{~cm}$ behind the front wall of the conditioning chamber (where the food magazine was located) and was used to present a white noise stimulus (produced by a Grason-Stadler white noise generator). The white noise measured $12 \mathrm{~dB}$ above a background level of $78 \mathrm{~dB}(\mathrm{C}$ weighting). The chamber was dark, except when the visual stimulus was presented. A fan attached to the outer shell provided for crossventilation within the shell, as well as background noise. All experimental events were controlled and recorded automatically by a 386 IBM-clone microcomputer and interfacing equipment (Alpha Products). 


\section{Procedure}

Prior to the beginning of this experiment, the rats were magazine trained with pellet and sucrose reinforcers. On each of 2 days, one magazine training session with 1 reinforcer was followed immediately by a second session with the alternative reinforcer. In each of these sessions, 20 reinforcers of one kind were delivered according to a variable time 60 -sec schedule. Sucrose $(0.2 \mathrm{ml}$ of a $24 \%$ solution $)$ and pellet (a single 45-mg pellet) reinforcers were delivered to the same food magazine.

Instrumental training. On the day following magazine training, all the rats were taught first to press the lever for one outcome and then to pull the chain for the other outcome. This training ceased after each response was reinforced 75 times. For half of the rats, leverpress responses were reinforced by pellets, and chain pull responses were reinforced by sucrose, and for the remaining rats, these responseoutcome contingencies were reversed. Instrumental training continued with variable interval (VI) schedules of reinforcement for a total of eight sessions. The average VI value increased in Sessions 1-3 from 10 to 20 to $30 \mathrm{sec}$, and it was $60 \mathrm{sec}$ in Sessions 4-8. The rats were given two 20-min sessions per day, one for the lever and one for the chain. Only one manipulandum was present in a session, and the order of training with lever and chain was balanced across days.

Pavlovian backward conditioning. The rats received backward conditioning with the light (L) and noise (N) stimuli over the next 16 sessions. The chain was withdrawn from the chamber and the lever made inaccessible by the sheet metal covering during these sessions. Each backward conditioning session was approximately $35 \mathrm{~min}$ in duration and included two 30 -sec presentations each of $\mathrm{N}$ and $\mathrm{L}$. Four different trial sequences (NLLN, LNNL, NLNL, and LNLN) were used irregularly across sessions. The intertrial intervals (CS offset to the next US onset) averaged 8 min, with a range between 5.5 and $10.5 \mathrm{~min}$. On a given backward conditioning trial, the CS was presented $10 \mathrm{sec}$ after the US had been presented. This interval was chosen on the basis of informal observations that rats usually consume the USs within approximately $5 \mathrm{sec}$ but, occasionally, may take up to $10 \mathrm{sec}$. Thus, with these parameters, the rats were very likely to have experienced truly backward US-CS pairings. For half of the subjects from each of the two counterbalanced groups of the instrumental training phase, pellets were paired with $\mathrm{N}$ and sucrose with $\mathrm{L}$, whereas the reverse was true for the remaining subjects.

Pavlovian-to-instrumental transfer test. One instrumental retraining session on each of the lever and the chain was given to the rats on the day after the last day of backward conditioning. The transfer test occurred on the following day. In this test, the lever and the chain were concurrently available during the entire session, but no reinforcements could be earned. Following a 1-min warm-up, $\mathrm{N}$ and $\mathrm{L}$ each were presented 16 times in the following sequence: NLLNLNNLNNLLNLNLLNLNLLNNLNNLNLLN. Each stimulus was $30 \mathrm{sec}$ long, and the stimuli were separated by a 30 -sec intertrial interval.

\section{Statistical Analysis}

Analysis of variance (ANOVA) techniques were used to evaluate the data. The post hoc methods of Rodger $(1974,1975)$ were used to further evaluate the source of the simple main effects and interactions for the acquisition data presented in Figure 1. According to this method, Type I error rate is controlled per contrast, and the value we adopted was .05 .

\section{Results}

Instrumental training was successful. On the final day of instrumental VI training, the rats were responding at a mean rate of 10.3 responses per minute (combined across manipulanda and reinforcer type). The instrumental response rates broken down by reinforcer and response type

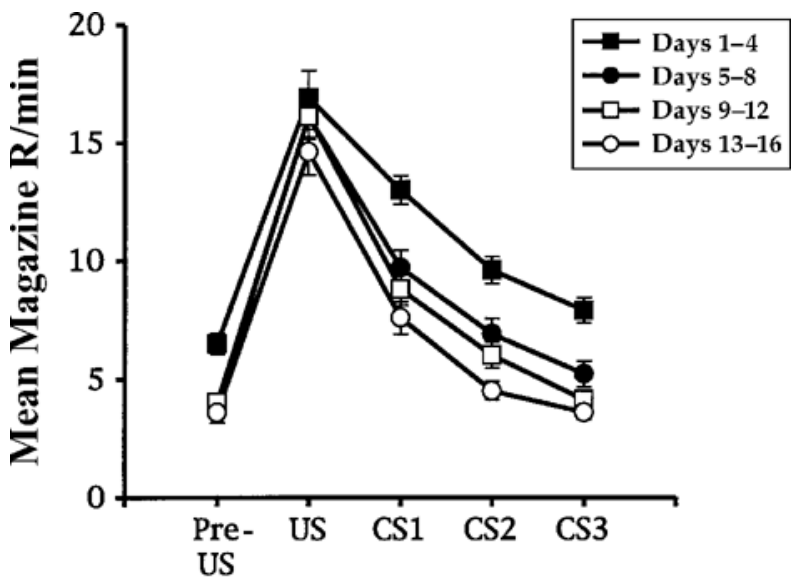

\section{0-sec Intervals}

Figure 1. Mean $( \pm S E)$ magazine responses/minute in the 30sec pretrial interval and in successive 10 -sec intervals post-US and continuing across the back ward US-CS trial. The distribution of responding across the trial is displayed in successive foursession blocks of backward training.

revealed more leverpress than chain-pull responses (10.9 and 9.6 responses per minute, respectively) and more responses reinforced by pellets than by sucrose (11.4 and 9.3 responses per minute, respectively). These differences did not interact with the data of primary interest from the transfer test session, reported below.

\section{Pavlovian Backward Conditioning}

The magazine data were examined during backwardconditioning sessions in order to determine whether the backward procedure we used in fact produced backward pairings between consumption of the USs and presentation of the CSs. On each trial, magazine response rates were recorded for the 30 -sec interval prior to the delivery of the US, during the 10-sec interval after US delivery (the interval in which it was expected that the rat would consume the US), and during each of three successive 10-sec intervals (beginning, middle, and end) of the CS. These data are depicted over four-session blocks of training in Figure 1. They illustrate that magazine responding was concentrated in the 10-sec interval immediately following US delivery but that responding persisted to some extent into the CS. Moreover, with extended training, this pattern of magazine response sharpened, with less responding occurring during the CS intervals. The latter fact probably reflects a greater efficiency in consuming the USs with extended training. But because these data likely reflect some combination of unconditioned responses and conditioned responses (CRs), they are important merely in documenting that the backward training procedure we used was successful at producing backward pairings between US consumption and CS.

A separate interval $\times$ block $\times$ replication ANOVA performed on these data confirmed these impressions. The 


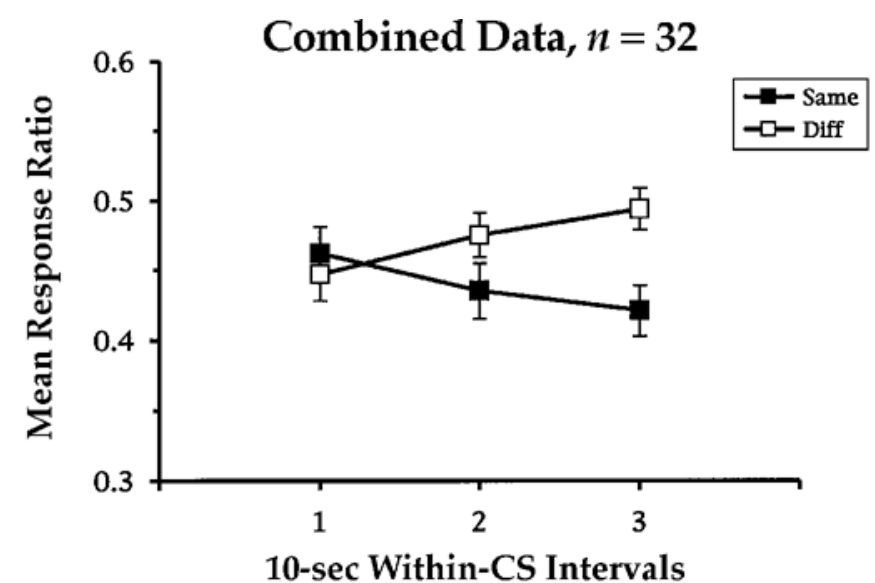

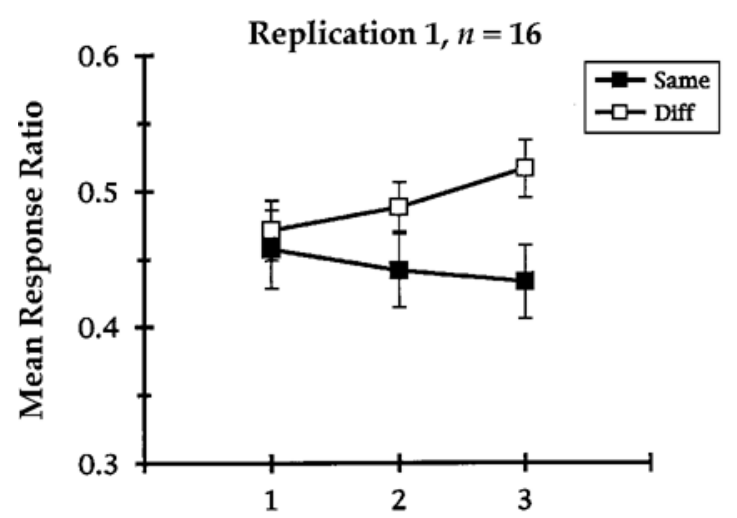

10-sec Within-CS Intervals

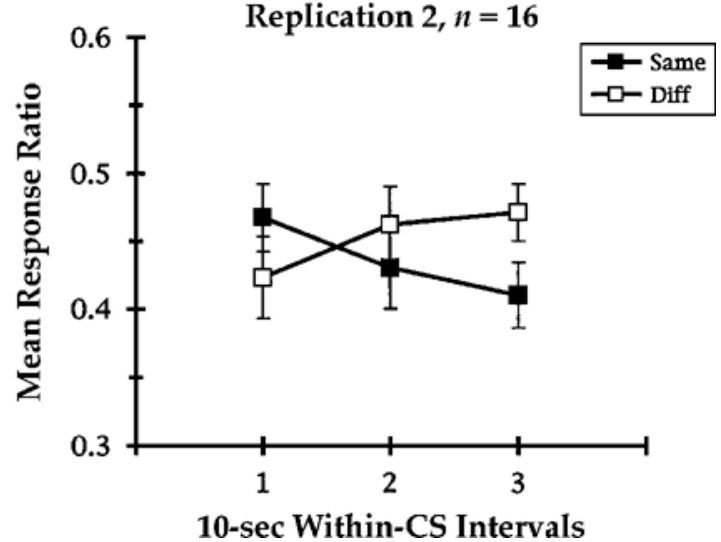

10-sec Within-CS Intervals

Figure 2. Mean $( \pm S E)$ instrumental responding across the stimulus in the transfer test when the backward conditioned stimulus (CS) and the instrumental response were trained with the same or different outcomes. Response rates during the stimulus were conditionalized upon prestimulus response rates.

main effects of interval $[F(4,120)=117.04]$ and block $[F(3,90)=53.80]$ were both significant, as was the interval $\times$ block interaction $[F(12,360)=2.94]$. There were no main effects or interactions involving the replication factor. Separate one-way ANOVAs examining the block effect revealed differences at each interval $\left(F_{\mathrm{s}}>4.94\right)$. Post hoc tests indicated that responding was higher in the first block than in any other block of training in every interval except the US interval. Responding during the final block of training was lower than that in every other block during the US interval and the first two CS intervals. This pattern of responding reflects an increasing concentration of magazine responding around the US interval as training progressed.

\section{Pavlovian-to-Instrumental Transfer Test}

The transfer test results are presented in Figure 2. The data are presented separately for instrumental responses that had been reinforced earlier with the same outcome as that paired with the CSs or with the different outcome. Since there were no interactions involving the stimuli $(\mathrm{N}$ vs. $\mathrm{L}$ or pellet-paired vs. sucrose-paired stimuli), the data have been collapsed across these stimulus factors. The data are displayed collapsed over replications (top), as well as separately for each replication (below), in order to illustrate the stability of the effect over replications. Finally, the instrumental response rate data have been transformed to response ratios of the form $a /(a+b)$, where $a$ denotes responding during the CS and $b$ denotes responding in the pre-CS interval. There were no differences in mean pre-CS same (4.4 responses per minute) and different response rates (4.4 responses per minute).

The data indicate a small suppression of both sameoutcome and different-outcome responses early in the CS but an increasing suppression of same-outcome responses and a decreasing suppression of different-outcome responses across the CS. These data were evaluated with a replication (first or second) $\times$ response (same or different outcome) $\times$ interval (first, second, or third 10-sec interval) $\times$ stimulus (pellet associated or sucrose associated) ANOVA. The only significant effect in this analysis was the response $\times$ interval interaction $[F(2,62)=7.02]$. Subsequent simple main effects tests determined that differentoutcome response ratios were greater than same-outcome 
response ratios in the third interval only $[F(1,93)=7.98]$. Similar patterns of responding across the CS were apparent in both replications, and there were no interactions involving this factor.

\section{Discussion}

The data from both replications of the present study were consistent in demonstrating that backward-trained CSs exert an outcome-specific effect upon instrumental choice. Of special interest is the fact that this effect is exactly opposite to that seen in prior research with forward-trained CSs. In particular, whereas forward-trained CSs have been shown to selectively increase the rate of instrumental responses with which they share an outcome (e.g., Colwill \& Rescorla, 1988; Kruse et al., 1983), the present results document that backward trained CSs selectively decrease the rate of instrumental responses with which they share an outcome. The most straightforward interpretation of these two sets of findings is that forward conditioning results in the formation of outcome-specific excitatory associations between the CS and the US and backward conditioning results in the formation of outcome-specific inhibitory associations.

However, before accepting such a conclusion, an alternative way of conceptualizing these data should be considered. Cole and Miller (1999) have applied their temporalcoding hypothesis to the case of backward conditioning One element of this hypothesis is that backward US-CS pairings result in the formation of an excitatory association between the two stimuli (see also Barnet \& Miller, 1996; Williams \& Hurlburt, 2000). A second element of the temporal coding hypothesis is that information regarding the temporal aspects of the backward procedure (namely, the stimulus duration and temporal order of the two events) is assumed to be part of what is learned. Cole and Miller described this aspect of backward learning in two ways. First, they discussed it in terms of a temporal maplike representation of the backward conditioning sequence. In essence, the temporal coding hypothesis asserts that when a well-trained backward CS is presented, it is assumed to evoke a representation that codes the US occurring prior to the CS. Second, Cole and Miller described the welltrained backward CS as a signal for the future absence of the US. Either of these relationships could result in the CS's acquiring what Cole and Miller refer to as "negative signal value," which they assume is responsible for inhibitory performance. If this negative signal value were assumed to be outcome specific, the present results could be explained by the temporal coding hypothesis, as well as in terms of inhibitory CS-US associations. The next experiment was designed to provide some information bearing on the temporal coding account of Experiment 1.

\section{EXPERIMENT 2}

The present experiment had two aims. We sought to provide additional information relevant to the temporal coding hypothesis and to provide additional evidence relating to the claim that backward conditioning results in the CS's acquiring sensory-specific inhibitory control (however one might wish to interpret that control).

We further explored the temporal coding hypothesis by asking whether the backward or the forward temporal information provided by the backward CS is more important in determining negative signal value. According to temporal coding theory, after a large number of trials a backward conditioned stimulus evokes a representation of the US as having just occurred. That is why, in Cole and Miller(1999), the backward conditioned stimulus produced excitatory second-order conditioning to another stimulus that occurred just before it. In that case, the representation of the US evoked in temporal coding is acting just like presentation of the US, which would also produce excitatory conditioning. If the same aspect of temporal coding is responsible for the outcome-specific inhibitory effect of the backward conditioned stimulus in the choice test of Experiment 1 , one would expect the noncontingent presentation of the US to also have an outcome-specific inhibitory effect on choice.

A different expectation develops if we assume that the CS acquires negative signal value because it signals the future omission of the US, and not because of its backward relation to the US. If this were the case, there would be little reason to suppose that a signal for the omission of the US and the noncontingent presentation of the US should influence instrumental choice in the same way. Indeed, if the USs in our experiment acquired outcome-specific discriminative properties during instrumental training, when only one response and reinforcer could occur in a given session (e.g., Rescorla \& Skucy, 1969), we should expect noncontingent USs to exert selective excitatory control over the instrumental responses that they previously reinforced.

In the present experiment, subjects that served in the second replication of Experiment 1 were given additional instrumental retraining sessions before being exposed to transfer-of-control tests in which the USs were presented noncontingently. Instrumental choice was measured in these tests in successive intervals following delivery of the US. If a negative signal value acquired by a backward CS is derived from the information it conveys about the prior occurrence of the US, we would expect the actual occurrence of the US to similarly suppress, in post-US intervals, the instrumental response that was previously reinforced by that US. We would not expect this result if the CS's negative signal value depended on signaling the future absence of the US.

Following these noncontingentUS tests, the subjects were administered an additional test to assess the sensory specificity of learning during backward conditioning. For this purpose, an outcome-specific retardation-of-acquisition test was run (Pearce, Montgomery, \& Dickinson, 1981). Before this test, the subjects first were given a minimal amount of additional backward training, and then they were segregated into two separate subgroups $(n=8)$, each of which was subsequently given forward conditioning 
with the CSs and USs used previously. One of these two subgroups, Group Same, was given forward training with the same CS-US combinations that they had earlier encountered during backward conditioning. The other subgroup, Group Reverse, was given forward training with the CS-US combinations reversed between the backward and the forward conditioning phases.

This comparison can be thought of as a kind of retardation test for conditioned inhibition. It was hypothesized that if backward conditioning results in outcome-specific inhibitory associations, it would be difficult to train the CSs as excitors when they served as forward signals for the very USs with which they previously had been paired backwardly. In other words, the acquisition of magazine approach responding in Group Same would be expected to be slower than acquisition in Group Reverse. Note that this comparison is better suited than most comparisons for identifying an outcome-specific effect of backward training, because it avoids the problems inherent in any design in which two groups receive different treatments in Phase 1. In the present case, both subgroups were exposed to backward and then forward conditioning procedures, with the only difference being the specific CS-US combinations in the two phases. Therefore, any difference between the two groups during the forward training phase would have to be attributed to the different CS-US combinations they experienced in the two phases. This could matter only if sensory-specific learning occurs in both phases.

\section{Method}

Subjects

Only the subjects from the second replication of Experiment 1 were used in Experiment 2, and they were maintained in the same manner as they had been previously.

\section{Apparatus}

The same apparatus as that in Experiment 1 was used.

\section{Procedure}

US tests. Beginning 1 week after the Pavlovian-to-instrumental transfer test of Experiment 1, the subjects in the second replication were administered three US test sessions. Each of these was preceded by two instrumental retraining sessions (as has been described previously). In the first two US test sessions, one or the other outcome was delivered 16 times, noncontingently, at 65 -sec intervals starting after the first minute. The rats had continuous access to the lever and the chain, and response rates were recorded in each of six, 10 -sec post-US intervals beginning $5 \mathrm{sec}$ after US delivery. Half of these subjects received pellets in Test 1 and sucrose in Test 2, whereas the remaining subjects were given the reverse order. Both pellets and sucrose were delivered noncontingently in US Test 3 . However, in this test, the interreinforcement interval was increased to $185 \mathrm{sec}$ to achieve greater separation between the effects of successive noncontingent outcomes, and there were six presentations each of pellet and sucrose in the following order: PSPSSPPSSPSP No responsecontingent reinforcers were presented during these tests.

Pavlovian retardation test. Six days after US Test 3, the subjects were given two additional backward conditioning retraining sessions (as a reminder of the backward contingencies they had experienced in Experiment 1). Then these subjects were exposed to 24 sessions of forward conditioning with both $\mathrm{N}$ and $\mathrm{L}$. The subjects were first segregated into two subgroups $(n=8)$ that were matched in terms of their performance during the Pavlovian-to-instrumental transfer test (Experiment 1), as well as the US tests. In addition, all of the above-mentioned counterbalancing was preserved equally in each of these subgroups. One of these subgroups, Group Same, was exposed to forward conditioning trials in which $\mathrm{N}$ and $\mathrm{L}$ preceded the USs that they had previously followed. For the other subgroup, Group Reverse, N and L preceded the USs with which they had not been paired during backward conditioning. Each of these forward conditioning sessions was identical to the backward conditioning sessions, with the exception that the USs were presented immediately after the termination of the CSs.

\section{Results}

\section{US Tests}

The results of the US tests are displayed in Figure 3. The data are expressed in terms of response rates occurring in the 5-sec interval in which the US was presented, as well as in each of six, post-US intervals. During the first two US

\section{US1 \& US2 in Separate Sessions}

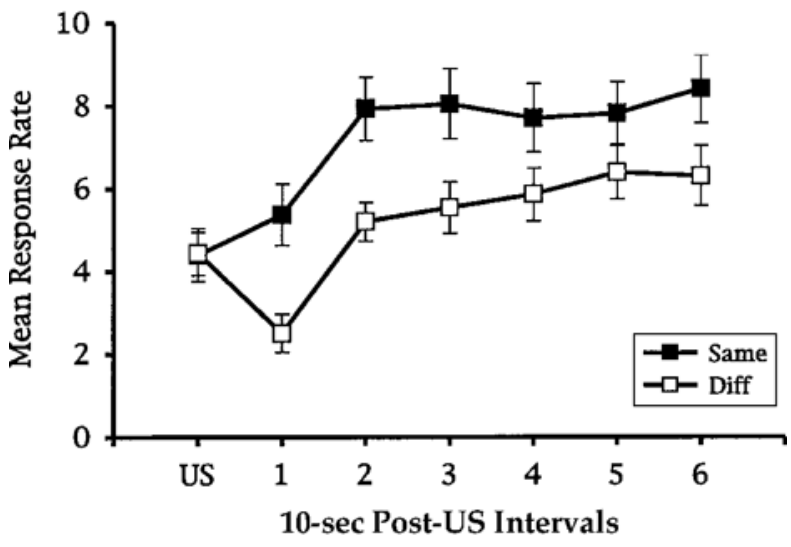

US1 \& US2 in Same Session

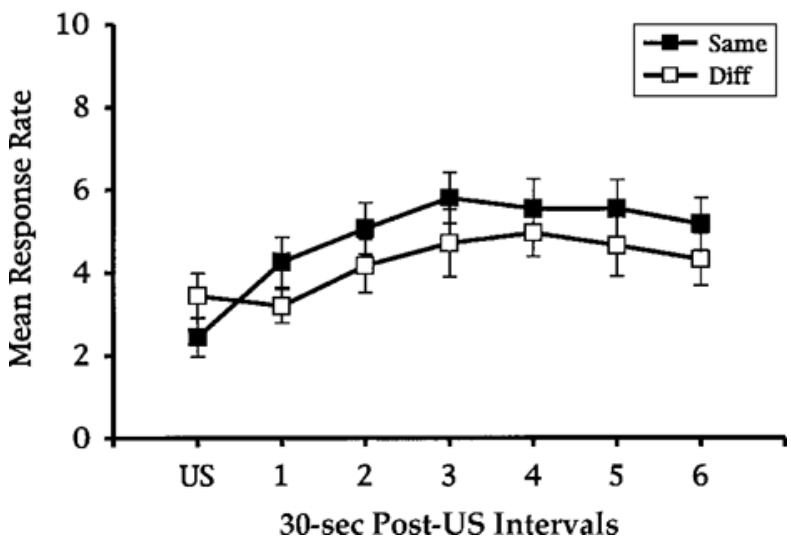

Figure 3. Mean $( \pm S E)$ instrumental responses/minute in the noncontingent unconditioned stimulus (US) tests. Each US first was tested in separate sessions (upper panel) with a 65 -sec interUS interval, and then the two USs were presented in the same test session with a 185-sec inter-US interval (lower panel). Responding is displayed during the interval in which the US was presented and in each of six separate post-US intervals, according to whether the response was reinforced previously by the noncontingent US (Same) or not (Diff). 
tests (upper panel, data combined across tests), pellet and sucrose reinforcers were presented noncontingentlyin different sessions. In the third US test (lower panel), pellet and sucrose reinforcers were presented in the same session. The results from the two types of US test sessions were very similar. Noncontingent presentations of a reinforcer elevated the instrumental response on which it earlier had been contingent more than it elevated the alternative response.

Separate response (same/different) $\times$ interval (postUS Intervals 1-6) ANOVAs were performed on the data depicted in each panel of Figure 3. The data for the intervals in which the US was presented were not analyzed, because it is likely that responding in this interval was suppressed by consumption of the US. For the separated US test sessions (upper panel), there were significant main effects of response $[F(1,15)=28.18]$ and interval $[F(5,75)=$ 20.71]. The response $X$ interval interaction was not significant. When both USs were presented in the same session (lower panel), there were also significant main effects of response $[F(1,15)=26.38]$ and interval $[F(5,75)=$ 8.24], but no interaction. The absolute size of the effect appears somewhat lower when both USs were presented in the same session (lower panel), as might be expected given that, on occasions in which the two USs alternated, the selective facilitative effect would have to overcome the opposite effect of the preceding trial. However, it is difficult to make anything of this impression, because the two types of sessions differed in duration, as well as in test order. The session in which both USs were presented was longer because the inter-US interval was lengthened in an effort to achieve greater separation between the effects of successive noncontingent outcomes. Because of this fact, more extinction in this session would have lowered overall response rates more than in the single US tests.

\section{Retardation of Acquisition Test}

Figure 4 displays, for Groups Same and Reverse, the mean rate of magazine responding over successive blocks of retardation testing (expressed as CS - pre-CS difference scores). The data were combined across both CSs. The figure illustrates that Group Reverse, the group trained with different outcomes in the backward and the forward conditioning phases, reached a higher rate of magazine approach responses than did Group Same.

A group $\times$ block ANOVA performed on these data revealed a significant main effect of block $[F(5,70)=10.25]$, as well as a significantgroup $\times$ block interaction $[F(5,70)=$ 2.94]. Subsequent between-groups ANOVAs conducted at each block indicated that the difference score was greater in Group Reverse than in Group Same in Block $6[F(1,33)=$ 4.72]. The groups did not differ in any other block.

A similar analysis applied to the pre-CS response rate data during the retardation test revealed a significant block effect $[F(5,70)=3.25]$ and a significant group $\times$ block interaction $[F(5,70)=3.03]$, in large part due to very low error variance with very low levels of responding in the pre-CS periods. However, subsequent between-

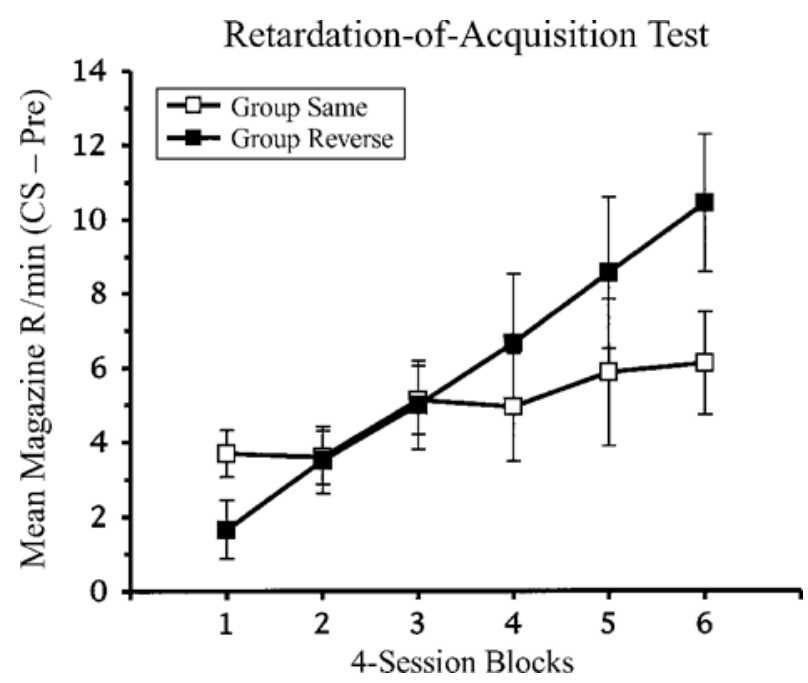

Figure 4. Mean $( \pm S E)$ magazine responses/minute are displayed in four-session blocks of the retardation-of-acquisition test in rats trained with the same (Group Same) or reversed (Group Reverse) stimulus-outcome assignments in the backward- and the forward-conditioning phases. Response rates are shown for the conditioned stimuli (CSs), combined, in the form of CS - preCS difference scores.

groups ANOVAs at each block failed to indicate that the groups differed in any block [largest $F(1,24)=1.62]$. The pre-CS means for Group Reverse and Group Same on Block 6, respectively, were 1.8 and 2.4 responses per minute.

\section{Discussion}

The present study had two objectives. First, we further explored predictions arising from the temporal coding hypothesis by asking whether the backward or the forward temporal information provided by the backward CS is more important in determining negative signal value. Second, the experiment provided additional evidence relating to the possibility that backward conditioning results in sensory-specific inhibitory control.

This experiment demonstrated slower acquisition of conditioned magazine approach responses when the CS was given forward pairings with the same US with which it previously had been given backward pairings, as opposed to a different US. This result is consistent with the claim that sensory-specific inhibitory control is established during backward conditioning.

In addition, the results indicate that noncontingent US presentations exert effects on instrumental choice that are opposite to the influence of a backward CS. This result has implications for Cole and Miller's (1999) temporal coding hypothesis. Above, we noted that according to this hypothesis, negative signal value in a backward CS can be thought of in two ways. The backward CS may be coded as providing temporal information (1) about the prior occurrence of the US or (2) about the future absence of the US. We reasoned that if negative signal value in the backward CS is related to the information it conveys about the 
prior occurrence of the US, the actual occurrence of the US should exert a similar effect on instrumental choice. The results show that the USs clearly did not exert an effect similar to the effect the CSs had in Experiment 1. This suggests that if negative signal value determined performance to the backward CSs in Experiment 1, it was information about the future absence of the US, and not about the prior occurrence of the US, that was important in governing instrumental choice.

Indeed, in the present experiment, the noncontingentUSs controlled instrumental choice in a manner that was consistent with their discriminative stimulus properties presumed to have been acquired during the instrumental training phase (e.g., Rescorla \& Skucy, 1969). The responses were trained in separate sessions during that phase, and this could have allowed each US to acquire control over the instrumental response that it reinforced. The new feature of the present data is the finding that USs differing in their qualitative, but presumably not their motivational, properties also can acquire selective discriminative control (see also Trapold \& Overmier, 1972).

\section{GENERAL DISCUSSION}

The data from the present experiments suggest that outcome-specific associations are learned as a result of backward US-CS pairings. Moreover, the transfer and the retardation test data are consistent with the view that these outcome-specific associations are inhibitory. In the latter portion of the CS, instrumental choice was biased away from the response with which the CS shared an outcome. Also, CR acquisition was more rapid when the CSs were paired with different USs in the backward and forward phases, as compared with when they were paired with the same US in the two phases. An additional noteworthy feature of the present data is that backward-trained CSs and the USs with which they were paired had opposite effects on instrumental choice.

The present data are quite clearly in line with predictions derived from AESOP theory (Wagner \& Brandon, 1989). As was noted above, this theory assumes that outcomespecific excitatory or inhibitory associations could result from backward US-CS pairings, depending on the USCS interval and the decay rates assumed for sensory processing of the US. Prior to running these experiments, we had no a priori reason to suspect that the US-CS interval we chose would be more favorable for sensory-specific excitatory or inhibitory associations' being learned. However, the results imply that the 10-sec US-CS interval used here allowed for a substantial amount of decay of sensory processing of the US into its A2 state at the time the CS was presented. This would have meant that A1 processing of the CS co-occurred with A2 processing of the sensory features of the US, a condition required for sensory-specific inhibitory learning. This perspective makes sense of both the transfer results and the retardation test results. Choice in favor of the instrumental response with which the CS did not share an outcome could reflect the CS's inhibitory effect on the outcome representation activated by the instrumental response with which it did share an outcome. Furthermore, the observation of slower CR acquisition in Group Same can be interpreted similarly in terms of outcomespecific conditioned inhibition. Importantly, if one interprets the transfer test as a kind of summation test for inhibition, the present experiment documents for the first time, using both of the commonly accepted standards for inhibition (Rescorla, 1969), that backward conditioning results in outcome-specific conditioned inhibition.

The present data are also relevant to other theories of backward conditioning. For instance, Cole and Miller's (1999) temporal coding hypothesis assumes that a backward CS acquires an excitatory association with the US, as well as a negative signal value. Furthermore, a negative signal value is assumed to be derived from temporal information the CS conveys about the prior occurrence and/or the future nonoccurrence of the US. This temporal information, in turn, is assumed to determine whether the CS will exert excitatory or inhibitory control over conditioned responding. For this approach to account for our Pavlovian-to-instrumental transfer test results, it must be assumed that (1) the backward CSs acquired negative signal value that was derived from temporal information the CSs conveyed about the future absence, rather than the prior occurrence, of the USs and (2) the negative signal value was outcome specific. The first of these assumptions seems warranted (as was discussed above) by the finding that CSs and USs had opposing effects on instrumental choice. The second of these assumptions requires some additional comment. Since, in the present experiments, the backward CSs were each followed by a minimum 5.5min interval in which neither US was presented, there is reason for thinking that each CS may well have signaled the absence of both USs. It was also true, however, that in these experiments, the USs were more likely to alternate than to repeat across trials. Thus, after each CS, there was a shorter interval to the occurrence of the alternate US than to the US that the CS had followed. Therefore, for the backward CS to have acquired outcome-specific negative signal value on the basis of predicting a period free of the US, the rats must have been sensitive to the greater average time to the next US that was the same US than to the next US that was different.

An alternative way of conceptualizing backward conditioning arises from the behavior systems theory (e.g., Silva \& Timberlake, 2000; Silva et al., 1998). According to this view, with relatively short interstimulus intervals, backward US-CS pairings should result in subjects' associating the CS with a behavioral mode (focal search) that is similar (although not identical) to the focal search mode assumed to be engaged by forward pairings. This description suggests that forward and backward conditioning ought to produce similar learning when the focal search mode is equally engaged. The magazine entry data collected during backward conditioning (see Figure 1) indicate that magazine responding persisted for some time following the $10-$ sec interval during which the US was presented 
(and consumed). On the basis of this, we expected the CS to be associated with postfood focal search responses (that included magazine entry) engaged by that specific US, much as would be a CS from forward conditioning. The transfer data are inconsistent with this expectation. Clearly, in the context of behavior systems theory, the present data would suggest that sometimes prefood and postfood focal search modes are strikingly different.

Although the present experiments clearly document that backward conditioning can result in outcome-specific inhibitory effects, there are several empirical issues that remain unresolved. Three parameters shown to be important in backward conditioning include the extent of training (Cole \& Miller, 1999; Heth, 1976), the US-CS interval (e.g., Maier, Rapaport, \& Wheatley, 1976), and the intertrial interval (e.g., Moscovitch \& LoLordo, 1968). One especially optimal set of conditions for generating inhibitory conditioning includes extended training with short US-CS intervals and long intertrial intervals (Moscovitch \& LoLordo, 1968). Parametric explorations of these variables may establish important boundary conditions for obtaining outcome-specific conditioned inhibition in backward conditioning.

Another important empirical issue concerns the comparison of different inhibitory conditioning procedures. For example, although prior research has documented the existence of outcome-specific inhibitory control by discriminative stimuli in instrumental settings (e.g., Bonardi, 1989; Colwill, 1991), there is relatively little evidence for outcome-specific inhibition from Pavlovian procedures (LoLordo \& Fairless, 1985). Moreover, Pavlovian studies in which this issue has been examined have not assessed backward conditioning, which may differ from other inhibitory conditioning procedures in its ability to support outcome-specific inhibitory learning.

Three studies have assessed the outcome specificity of inhibition resulting from inhibitory conditioning procedures other than backward conditioning. LoLordo (1967) superimposed a Pavlovian differential conditioning procedure in which the US was either a footshock or a loud noise upon an unsignaled footshock avoidance baseline. The CS - for shock markedly suppressed dogs' avoidance behavior, but the $\mathrm{CS}$ - for loud noise did not produce significant suppression, although there was a trend in that direction. This result constituted weak support for outcome specificity of inhibition; however, two other studies in which aversive conditioning was used provided no support for specificity. Pearce et al. (1981) found that following conditioned inhibition training $(\mathrm{A}+, \mathrm{AX}-)$ in the eyeblink conditioning procedure, rabbits showed roughly equivalent retardation of excitatory conditioning, relative to controls, whether the inhibitor was repeatedly paired with the same para-orbital shock used in conditioning or with shock to the other eye. There was no evidence for outcome specificity. Nieto (1984) used a between-sessions negative correlation procedure, in which only CSs occurred in some sessions and only USs in others, to generate inhibition. For one group of rats, the US was loud noise, and for another, it was footshock. Then half the rats in each group received pairings of a second CS with footshock, and the others received pairings of that CS with loud noise. In a subsequent summation test, the putative inhibitor had the same inhibitory effect whether the second CS with which it was compounded had signaled footshock or the loud noise. Another experiment in the same report, using the conditioned inhibition procedure and both retardation-of-acquisition and summation tests, corroborated this finding. Again, there was no evidence for outcome-specific inhibition.

Like the present research, a study by Kruse et al. (1983) used appetitive Pavlovian conditioning and an instrumental choice test procedure to study outcome-specificinhibitionin that case, the outcome specificity of conditioned inhibition evoked by a CS that had been explicitly unpaired with the US. These authors first trained rats on a conditional discrimination task with differential outcomes. In the presence of one discriminative stimulus (S1), one instrumental response (R1) produced one positive outcome (O1), but a second instrumental response (R2) was not reinforced. In the presence of a second discriminative stimulus (S2), R2 produced a second positive outcome (O2), but $\mathrm{R} 1$ was not reinforced. The rats then received a Pavlovian inhibitory conditioning procedure in which CS - was explicitly unpaired with one of these outcomes. Outcomespecific inhibitory control by the CS- was then assessed in transfer tests with the CS tested by itself and in combination with each of the discriminative stimuli (S1 and S2). These tests revealed that $\mathrm{CS}-$ exerted an outcome-specific effect on the latency to complete the instrumental response requirement in the presence of the discriminative stimuli but, unlike in the present study, had no selective effect on choice. An important variable in detecting outcome-specific effects with this procedure is likely to be whether the instrumental baseline is free operant, as in the present study, or discriminated operant, as in Kruse et al. (1983).

Finally, there is one additional aspect of the present results that requires further investigation. The inhibitory Pavlovian-to-instrumental transfer effect we report was seen only in the last 10 -sec portion of the CS. This result may have a relatively simple explanation, or it may point to the involvement of more complex processes than have been considered thus far. For instance, one explanation rests on the assumption that the CS or, more likely, the no$U S$ representation that it presumably activates must be recruited for some minimal amount of time before behavioral effects can be observed. This assumption would make sense of the observation that the effect appears to be graded across the CS interval (see Figure 2). A somewhat more complex approach would be to assume that different portions of the CS are discriminably different. This would allow for different temporal components of the CS to be learned about differently. In particular, it may be assumedfor example, in line with AESOP - that the latter portion of the CS was in a more favorable position than the earlier portions to acquire conditioned inhibition. Precisely this sort of argument was made by Romaniuk and Williams 
(2000) in explaining why their 30-sec backward CS functioned as a conditioned inhibitor when tested in its entirety, although the first 3 -sec portion of the CS functioned as a conditioned excitor when tested separately. Regardless of which of these views is correct, it seems clear that a complete account of backward conditioning ultimately will have to take this temporal variable into account.

In summary, the present experiments document that backward conditioning results in the development of outcomespecific inhibitory control by the CS. This conclusion is substantiated by the results from Pavlovian-to-instrumental transfer and retardation-of-learning tests. Moreover, separate tests of noncontingentUSs during instrumental choice revealed that backward-trained CSs and USs had opposing effects on instrumental responding. The results should help to constrain further developments of existing theories of backward conditioning.

\section{REFERENCES}

BARnet, R. C., \& Miller, R. R. (1996). Second-order excitation mediated by a backward conditioned inhibitor. Journal of Experimental Psychology: Animal Behavior Processes, 22, 279-296.

Betts, S. L., Brandon, S. E., \& Wagner, A. R. (1996). Dissociation of the blocking of conditioned eyeblink and conditioned fear following a shift in US locus. Animal Learning \& Behavior, 24, 459-470.

BONARDI, C. (1989). Inhibitory discriminative control is specific to both the response and the reinforcer. Quarterly Journal of Experimental Psychology, 41B, 225-242.

Cole, R. P., \& Miller, R. R. (1999). Conditioned excitation and conditioned inhibition acquired through backward conditioning. Learning \& Motivation, 30, 129-156.

Colwill, R. M. (1991). Negative discriminative stimuli provide information about the identity of omitted response-contingent outcomes. Animal Learning \& Behavior, 19, 326-336.

Colwill, R. M., \& Motzkin, D. K. (1994). Encoding of the unconditioned stimulus in Pavlovian conditioning. Animal Learning \& Behavior, 22, 384-394.

Colwill, R. M., \& Rescorla, R. A. (1988). Associations between the discriminative stimulus and the reinforcer in instrumental learning. Journal of Experimental Psychology: Animal Behavior Processes, 14, 155-164.

Delamater, A. R. (1995). Outcome-selective effects of intertrial reinforcement in a Pavlovian appetitive conditioning paradigm with rats Animal Learning \& Behavior, 23, 31-39.

Delamater, A. R. (1996). Effects of several extinction treatments upon the integrity of Pavlovian stimulus-outcome associations. Animal Learning \& Behavior, 24, 437-449.

Delamater, A. R. (1997). Selective reinstatement of stimulus-outcome associations. Animal Learning \& Behavior, 25, 400-412.

Delamater, A. R, \& LoLordo, V. M. (1991). Event revaluation procedures and associative structures in Pavlovian conditioning. In L. Dachowski \& C. F. Flaherty (Eds.), Current topics in animal learning: Brain, emotion, and cognition (pp. 55-94). Hillsdale, NJ: Erlbaum.

HeTH, C. D. (1976). Simultaneous and backward fear conditioning as a function of number of CS-US pairings. Journal of Experimental Psychology: Animal Behavior Processes, 2, 117-129.

Kruse, J. M., Overmier, J. B., Konz, W. A., \& Rokke, E. (1983). Pavlovian conditioned stimulus effects upon instrumental choice behavior are reinforcer specific. Learning \& Motivation, 14, 165-181.

LoLordo, V. M. (1967). Similarity of conditioned fear responses based on different aversive events. Journal of Comparative \& Physiological Psychology, 64, 154-158.

LoLordo, V. M., \& FaIRLESS, J. L. (1985). Pavlovian conditioned inhibition: The literature since 1969. In R. R. Miller \& N. E. Spear (Eds.), Information processing in animals: Conditioned inhibition (pp. 1-50). Hillsdale, NJ: Erlbaum.

Maier, S. F., Rapaport, P., \& Wheatley, K. L. (1976). Conditioned inhibition and the UCS-CS interval. Animal Learning \& Behavior, 4, 217-220.

McNish, K. A., Betts, S. L., Brandon, S. E., \& Wagner, A. R. (1997). Divergence of conditioned eyeblink and conditioned fear in backward Pavlovian training. Animal Learning \& Behavior, 25, 43-52.

Moscovitch, A., \& LoLordo, V. M. (1968). Role of safety in the Pavlovian backward fear conditioning procedure. Journal of Comparative \& Physiological Psychology, 66, 673-678.

Nieto, J. (1984). Transfer of conditioned inhibition across different aversive reinforcers in the rat. Learning \& Motivation, 15, 37-57.

Pearce, J. M., Montgomery, A., \& Dickinson, A. (1981). Contralateral transfer of inhibitory and excitatory eyelid conditioning in the rabbit. Quarterly Journal of Experimental Psychology, 33B, 45-61.

Rescorla, R. A. (1969). Pavlovian conditioned inhibition. Psychological Bulletin, 72, 77-94.

Rescorla, R A. (1997). Spontaneous recovery after Pavlovian conditioning with multiple outcomes. Animal Learning \& Behavior, 25, 99-107.

Rescorla, R. A. (1999). Learning about qualitatively different outcomes during a blocking procedure. Animal Learning \& Behavior, 27, 140-151.

Rescorla, R. A., \& SKUCY, J. C. (1969). Effect of response-independent reinforcers during extinction. Journal of Comparative \& Physiological Psychology, 67, 381-389.

RoDGER, R. S. (1974). Multiple contrasts, factors, error rate, and power. British Journal of Mathematical \& Statistical Psychology, 27, 179-198.

RoDGER, R. S. (1975). The number of non-zero, post hoc contrasts from ANOVA and error-rate: I. British Journal of Mathematical \& Statistical Psychology, 28, 71-78.

Romaniuk, C. B., \& Williams, D. A. (2000). Conditioning across the duration of a backward conditioned stimulus. Journal of Experimental Psychology: Animal Behavior Processes, 26, 454-461.

Silva, F. J., \& Timberlake, W. (2000). A clarification on the nature of backward excitatory conditioning. Learning \& Motivation, 31, 67-80.

Silva, F. J., Timberlake, W., \& CeviK, M. O. (1998). A behavior systems approach to the expression of backward associations. Learning \& Motivation, 29, 1-22.

Tait, R. W., \& Saladin, M. E. (1986). Concurrent development of excitatory and inhibitory associations during backward conditioning. Animal Learning \& Behavior, 14, 133-137.

Trapold, M. A., \& Overmier, J. B. (1972). The second learning process in instrumental learning. In A. H. Black \& W. F. Prokasy (Eds.), Classical conditioning: II. Current research and theory (pp. 427-452). New York: Appleton-Century-Crofts.

WAGNER, A. R, \& BRANDON, S. E. (1989). Evolution of a structured connectionist model of Pavlovian conditioning (AESOP). In S. B. Klein \& R. R. Mowrer (Eds.), Contemporary learning theories: Pavlovian conditioning and the status of traditional learning theory (pp. 149189). Hillsdale, NJ: Erlbaum.

WatT, A. \& Honey, R. C. (1997). Combining CSs associated with the same or different USs. Quarterly Journal of Experimental Psychology, 50B, 350-367.

Williams, D. A., \& Hurlburt, J. L. (2000). Mechanisms of secondorder conditioning with a backward conditioned stimulus. Journal of Experimental Psychology: Animal Behavior Processes, 26, 340-351.

(Manuscript received October 10, 2002; revision accepted for publication July 21,2003 .) 